\title{
From the Weyl Anomaly to Entropy of Two-Dimensional Boundaries and Defects
}

\author{
Kristan Jensen, ${ }^{1, *}$ Andy O’Bannon, ${ }^{2, \dagger}$ Brandon Robinson, ${ }^{2, \$}$ and Ronnie Rodgers ${ }^{2, \S}$ \\ ${ }^{1}$ Department of Physics and Astronomy, San Francisco State University, San Francisco, California 94132, USA \\ ${ }^{2}$ STAG Research Centre, University of Southampton, Highfield, Southampton SO17 1BJ, United Kingdom
}

(Received 21 January 2019; published 19 June 2019)

\begin{abstract}
We study whether the relations between the Weyl anomaly, entanglement entropy (EE), and thermal entropy of a two-dimensional (2D) conformal field theory (CFT) extend to 2D boundaries of 3D CFTs, or 2D defects of $D \geq 3$ CFTs. The Weyl anomaly of a 2D boundary or defect defines two or three central charges, respectively. One of these, $b$, obeys a $c$ theorem, as in 2D CFT. For a 2D defect, we show that another, $d_{2}$, interpreted as the defect's "conformal dimension," must be non-negative if the averaged null energy condition holds in the presence of the defect. We show that the EE of a sphere centered on a planar defect has a logarithmic contribution from the defect fixed by $b$ and $d_{2}$. Using this and known holographic results, we compute $b$ and $d_{2}$ for 1/2-Bogomol'nyi-Prasad-Sommerfield surface operators in the maximally supersymmetric (SUSY) 4D and 6D CFTs. The results are consistent with $b$ 's $c$ theorem. Via free field and holographic examples we show that no universal "Cardy formula" relates the central charges to thermal entropy.

DOI: 10.1103/PhysRevLett.122.241602
\end{abstract}

Introduction.-CFTs play a central role in many branches of physics. In condensed matter physics, they describe critical points. In string theory, the worldsheet theory is a CFT. In quantum field theory, CFTs are fixed points of renormalization group (RG) flows.

CFTs in two-dimensional Minkowski space, i.e., 2D CFTs, enjoy Virasoro symmetry with central charge $c$. Unitarity plus ground state normalizability implies $c \geq 0$. For an RG flow from ultraviolet (UV) to infrared (IR) CFTs with central charges $c_{\mathrm{UV}}$ and $c_{\mathrm{IR}}$, respectively, unitarity, locality, and Poincare symmetry imply the $c$ theorem: $c_{\mathrm{UV}} \geq c_{\mathrm{IR}}$ [1]. These properties suggest $c$ measures the effective number of massless degrees of freedom (DOF), which is expected to be non-negative and to decrease along RG flows.

Virasoro symmetry also implies that $c$ determines at least four other following quantities that can count DOF: (i) $c$ fixes the normalization of the two-point function of the stress-energy tensor, $T^{\mu \nu}$. (ii) On a background with a nontrivial spacetime metric $g_{\mu \nu}$ with Ricci scalar $\mathcal{R}$, quantum effects can produce the Weyl anomaly, $T_{\mu}^{\mu}=-(c / 24 \pi) \mathcal{R}$ [2-5]. (iii) The EE of a spatial interval of length $\ell$, which measures the strength of vacuum correlations, is $S_{\mathrm{EE}}=$ $(c / 3) \ln (\ell / \varepsilon)+\mathcal{O}\left(\varepsilon^{0}\right)$ [6,7], with UV cutoff $0<\varepsilon \ll 1$. (iv)At nonzero temperature $T$ the CFT's entropy density, $s$,

Published by the American Physical Society under the terms of the Creative Commons Attribution 4.0 International license. Further distribution of this work must maintain attribution to the author(s) and the published article's title, journal citation, and DOI. Funded by SCOAP. which measures the number of thermodynamic microstates, is $s=(\pi / 6) c T[8,9]$.

While CFTs have an infinite correlation length, no real system is infinite: boundary conditions (BCs) will always be important. Moreover, no real system is perfect: defects such as impurities, domain walls between differently ordered phases, and so on will always be important. Constructing and classifying CFTs with conformally invariant boundaries (BCFTs) or defects (DCFTs) is thus crucial for describing an enormous number of systems.

In this Letter, we study a 2D boundary of a 3D CFT or 2D conformal defect in a $D \geq 3 \mathrm{CFT}$. We assume the boundary or defect is flat, i.e., a static straight line. Such a system does not have Virasoro symmetry in general: the 2D contribution to $T^{\mu \nu}$ is not conserved because energy and momentum can flow between the boundary or defect and the bulk CFT, and these systems have a finite number of symmetry generators following from the CFT's $S O(D, 2)$ conformal symmetry being broken to $S O(2,2) \times S O(D-2)$, where $S O(2,2)$ are conformal transformations leaving the static line invariant and $S O(D-2)$ are rotations about the static line [10].

We find that the logarithmic term in $S_{\mathrm{EE}}$ of a spherical region centered on a defect is fixed by $T_{\mu}^{\mu}$, while in general no simple relation exists between $T_{\mu}^{\mu}$ and $s$. The boundary or defect contribution to $T_{\mu}^{\mu}$ includes multiple central charges $[11,12]$. Assuming the averaged null energy condition (ANEC) holds in the presence of the defect, we conjecture a positivity bound on one of the defect central charges. In Ref. [13], we also find new central charges allowed in 4D if parity is broken. We use the method of Refs. $[21,22]$ to show that the logarithmic term in general depends on two defect central charges-confirming and 
extending a key result of Ref. [23]. Using this and known holographic results, we compute these central charges for certain 1/2-Bogomol'nyi-Prasad-Sommerfield (BPS) surface operators in the maximally SUSY 4D and 6D CFTs. Finally, for the free massless scalar and fermion 3D BCFTs and for 2D defects holographically dual to probe branes, we calculate $s \propto T$ at the boundary or defect, with no universal relation between the proportionality coefficient and central charges.

Conventions. - We start with a local, unitary, Lorentzian CFT on a $D \geq 3$ spacetime $\mathcal{M}$ with coordinates $x^{\mu}$ $(\mu=0,1, \ldots, D-1)$ and metric $g_{\mu \nu}$, which we will call the "bulk" CFT. We introduce a codimension $D-2$ defect along a static $2 \mathrm{D}$ submanifold $\Sigma$ with coordinates $y^{a}(a=0$, 1). We parametrize $\Sigma \hookrightarrow \mathcal{M}$ by embedding functions $X^{\mu}(y)$ such that $\Sigma$ 's induced metric is $\gamma_{a b} \equiv \partial_{a} X^{\mu} \partial_{b} X^{\nu} g_{\mu \nu}$. We denote $\mathcal{M}$ 's covariant derivative as $\nabla_{\mu}$ and $\Sigma$ 's induced covariant derivative as $\hat{\nabla}_{a}$, which acts on a mixed tensor $\mathcal{T}_{a}^{\mu}$ as $\hat{\nabla}_{a} \mathcal{T}_{b}^{\mu}=\partial_{a} \mathcal{T}_{b}^{\mu}+\Gamma_{\nu a}^{\mu} \mathcal{T}_{b}^{\nu}-\hat{\Gamma}_{a b}^{c} \mathcal{T}_{c}^{\mu}$. The second fundamental form is then $\Pi_{a b}^{\mu}=\hat{\nabla}_{a} \partial_{b} X^{\mu}$, with traceless part $\stackrel{\circ}{\Pi}_{a b}^{\mu} \equiv \Pi_{a b}^{\mu}-\frac{1}{2} \gamma_{a b} \gamma^{c d} \Pi_{c d}^{\mu}$

Physically, the defect can arise from 2D DOF coupled to the bulk CFT and/or BCs imposed on bulk CFT fields [24]. In 3D the defect is a domain wall between two CFTs, and if one of these is the "trivial" CFT, then the defect is a boundary.

The Weyl anomaly.-We denote the DCFT partition function as $Z$ and the generating functional of connected correlators $W \equiv-i \ln Z$, which are both functionals of $g_{\mu \nu}$ and $X^{\mu}$. Varying $W$, we define the stress-energy tensor, $T_{\mu \nu}$, and displacement operator, $\mathcal{D}_{\mu}$

$$
\delta W=\frac{1}{2} \int d^{D} x \sqrt{-g} \delta g_{\mu \nu}\left\langle T^{\mu \nu}\right\rangle+\int d^{2} y \sqrt{-\gamma} \delta X^{\mu}\left\langle\mathcal{D}_{\mu}\right\rangle,
$$

where $g \equiv \operatorname{det} g_{\mu \nu}$ and $\gamma \equiv \operatorname{det} \gamma_{a b}$. Invariance of $W$ under reparametrizations of $y^{a}$ implies $\mathcal{D}_{\mu}$ 's components along $\Sigma$ vanish [29]. Invariance of $W$ under reparametrizations of $x^{\mu}$ implies $\nabla_{\nu}\left\langle T^{\nu \mu}\right\rangle=-\delta^{D-2}\left\langle\mathcal{D}^{\mu}\right\rangle$, with $\delta^{D-2}$ a delta function that restricts to $\Sigma$ [29]. Physically, $\left\langle T^{\mu \nu}\right\rangle$ is not conserved at $\Sigma$ because the defect and bulk can exchange transverse energy momentum.

Our DCFTs are invariant under infinitesimal Weyl transformations, $\delta_{\omega} g_{\mu \nu}=2 \omega g_{\mu \nu}$ and $\delta_{\omega} X^{\mu}=0$, up to the Weyl anomaly [2-5]: $\delta_{\omega} W=\int d^{D} x \sqrt{-g} \omega\left\langle T_{\mu}^{\mu}\right\rangle$, where $\left\langle T_{\mu}^{\mu}\right\rangle$ is built from external sources, such as $g_{\mu \nu}$. We consider contributions to $\left\langle T_{\mu}^{\mu}\right\rangle$ from $g_{\mu \nu}$ and $\partial_{a} X^{\mu}$ only. Determining $\left\langle T_{\mu}^{\mu}\right\rangle$ 's most general form requires solving the WessZumino (WZ) consistency condition, $\left[\delta_{\omega_{1}}, \delta_{\omega_{2}}\right] W=0$, and then fixing any local counterterms that contribute to $\left\langle T_{\mu}^{\mu}\right\rangle$. In our DCFTs, $\left\langle T_{\mu}^{\mu}\right\rangle=\left\langle T_{\mu}^{\mu}\right\rangle_{\text {bulk }}+\delta^{D-2}\left\langle T_{\mu}^{\mu}\right\rangle_{\text {def }}$, where $\left\langle T_{\mu}^{\mu}\right\rangle_{\text {bulk }}$ and $\left\langle T_{\mu}^{\mu}\right\rangle_{\text {def }}$ are bulk CFT and defect Weyl anomalies, respectively, and we fixed local counterterms to cancel terms with normal derivatives of $\delta^{D-2}$. For $\left\langle T_{\mu}^{\mu}\right\rangle_{\text {bulk }}$ we will only need to know that $\left\langle T_{\mu}^{\mu}\right\rangle_{\text {bulk }}=0$ in odd $D$, but can be nonzero in even $D$, which defines the bulk central charge(s). For a $2 \mathrm{D}$ defect in a $D \geq 3$ DCFT $[11,12,30]$

$$
\left\langle T_{\mu}^{\mu}\right\rangle_{\mathrm{def}}=-\frac{1}{24 \pi}\left(b \mathcal{R}_{\Sigma}+d_{1} \stackrel{\circ}{\mathrm{II}}_{a b}^{\mu} \stackrel{\circ}{\mathrm{II}}_{\mu}^{a b}-d_{2} W_{a b}^{a b}\right),
$$

where $\mathcal{R}_{\Sigma}$ is $\Sigma$ 's intrinsic scalar curvature, $W_{a b c d}$ is the pullback of the bulk Weyl tensor to $\Sigma$, and $b, d_{1}$, and $d_{2}$ are defect central charges. When $D=3, W_{a b c d}=0$ identically, so $d_{2}$ exists only for $D \geq 4$.

Bounds on central charges. - As mentioned above, in a 2D CFT $c$ determines various observables and with reasonable assumptions, such as unitarity, obeys $c \geq 0$ and the $c$ theorem. By comparison, less is known about $b$, $d_{1}$, and $d_{2}$. Under Weyl transformations $\sqrt{-\gamma} \mathcal{R}_{\Sigma}$ changes by a total derivative, while both $\sqrt{-\gamma} \stackrel{\circ}{\mathrm{II}_{a b}^{\mu}} \stackrel{\circ}{\circ \mathrm{II}_{\mu}}$ and $\sqrt{-\gamma} W_{a b}^{a b}$ are invariant [4]. As a result, in the Euclidean DCFT on $\mathbb{S}^{D}$ of radius $r$ with bulk partition function $Z_{\mathrm{CFT}}$ and with defect along a maximal $\mathbb{S}^{2}, Z / Z_{\mathrm{CFT}} \propto(r \Lambda)^{b / 3}$ [29]. For a local, unitary defect RG flow, $b$ obeys a $c$ theorem, suggesting $b$ counts defect DOF [29]. WZ consistency forces $b$ to be independent of any marginal couplings. The normalization of $\mathcal{D}^{\mu}$ 's two-point function is fixed by $d_{1}$, such that unitarity implies $d_{1} \geq 0[31,32]$.

Table I shows $b, d_{1}$, and $d_{2}$ in the 3D BCFTs of a free, massless real scalar or Dirac fermion [29,33,34], and in CFTs holographically dual to Einstein gravity in $(D+1)$ dimensional anti-de Sitter space, $\mathrm{AdS}_{D+1}$, with metric $G_{M N}$ $(M, N=0,1, \ldots, D)$ and defect dual to a probe brane along $\mathrm{AdS}_{3}$ whose action $S_{\text {probe }}=-T_{\text {br }} \int d^{3} \xi \sqrt{-\operatorname{det}\left(P\left[G_{M N}\right]\right)}$ with tension $T_{\mathrm{br}}$ and brane coordinates $\xi$ [30]. In all of these theories, the central charges are $\geq 0$ with the exception of the scalar with Dirichlet BC, which has $b<0$. This example proves that unitarity does not require $b \geq 0$.

For a unitary 3D BCFT with unique stress-energy tensor at the boundary [35], Ref. [32] conjectured

$$
b=\frac{2 \pi^{2}}{3} \epsilon(1)-\frac{2}{3} d_{1},
$$

TABLE I. Central charges $b, d_{1}$, and $d_{2}$ of Eq. (1) for 3D BCFTs of free, massless real scalars with Dirichlet or Robin BC, or Dirac fermion with the unique conformal "mixed" BC $[29,33,34]$, and for a $2 \mathrm{D}$ defect dual to a probe brane of tension $T_{\text {br }}$ along $\mathrm{AdS}_{3}$ inside $\mathrm{AdS}_{D+1}$ of radius L [30].

\begin{tabular}{lcccc}
\hline \hline Theory & BC & $b$ & $d_{1}$ & $d_{2}$ \\
\hline Scalar & Dirichlet & $-1 / 16$ & $3 / 32$ & N/A \\
Scalar & Robin & $1 / 16$ & $3 / 32$ & N/A \\
Fermion & Mixed & 0 & $3 / 16$ & N/A \\
Probe brane & N/A & $6 \pi L^{3} T_{\mathrm{br}}$ & $6 \pi L^{3} T_{\mathrm{br}}$ & $6 \pi L^{3} T_{\mathrm{br}}$ \\
\hline \hline
\end{tabular}


where $\epsilon(v)$ is a contribution to $T^{\mu \nu}$ 's two-point function from exchange of spin-2 boundary operators, with $v \in$ $[0,1]$ the BCFT conformal cross ratio, with boundary at $v=1 \quad[31,36,37]$. Unitarity implies $\quad \epsilon(v) \geq 0$ [31]. However, if the BCFT has any spin-2 boundary operators of dimension $\Delta \in[2,3)$, then $\epsilon(v)$ diverges as $(1-v)^{\Delta-3}$ when $v \rightarrow 1$. In that case, unitarity does not constrain the sign of $\epsilon(1)$, the $(1-v)^{0}$ term in $\epsilon(v)$ 's expansion about $v=1$, and so $b$ has no definite lower bound. On the other hand, in the absence of such operators $\epsilon(v)$ is regular as $v \rightarrow 1$, unitarity implies $\epsilon(1) \geq 0$, and hence $b \geq-\frac{2}{3} d_{1}$. All the examples in Table. I obey this bound, and the free scalar with Dirichlet BC saturates it.

We can prove a new bound assuming the ANEC holds in the presence of the defect. The ANEC states that for any null direction $u, \int_{-\infty}^{\infty} d u\left\langle T_{u u}\right\rangle \geq 0$. Proofs of the ANEC for CFTs appear in Refs. $[38,39]$. Though these proofs have not yet been extended to BCFTs or DCFTs, they rely mainly on unitarity and causality, which in a BCFT or DCFT should suffice to guarantee that a lightlike observer's total energy is $\geq 0$.

While $S O(D, 2)$ symmetry forces $\left\langle T_{\mu \nu}\right\rangle=0$ for the undeformed CFT, the $S O(2,2) \times S O(D-2)$ symmetry preserved by $\Sigma$ allows $\left\langle T_{\mu \nu}\right\rangle \neq 0$. When $D=4$, Refs. [25,27] showed that by writing the most general form of $\left\langle T_{\mu \nu}\right\rangle$ such that it is well defined as a distribution and comparing its variation under constant Weyl transformations to the variation of $\left\langle T_{\mu}^{\mu}\right\rangle_{\text {def }}$ with respect to $g_{\mu \nu}$, $\left\langle T_{\mu \nu}\right\rangle$ completely determined by $d_{2}$. Generalizing to arbitrary $D \geq 4$ is straightforward: with coordinates $x^{i}$ transverse to $\Sigma(i=2,3, \ldots, D-1)$, and for a point a distance $\left|x^{i}\right|>0$ from $\Sigma$

$$
\begin{aligned}
\left\langle T^{a b}\right\rangle & =-\frac{h_{D}}{2 \pi} \frac{\eta^{a b}}{\left|x^{i}\right|^{D}}, \quad\left\langle T^{a i}\right\rangle=0, \\
\left\langle T^{i j}\right\rangle & =\frac{h_{D}}{2 \pi(D-3)} \frac{3 \delta^{i j}\left|x^{k}\right|^{2}-D x^{i} x^{j}}{\left|x^{l}\right|^{D+2}}, \\
h_{D} & \equiv \frac{1}{3 \operatorname{vol}\left(\mathbb{S}^{D-3}\right)} \frac{D-3}{D-1} d_{2},
\end{aligned}
$$

where $h_{D}$ is the defect's "conformal dimension" (see, e.g., Ref. [40]). Using $S O(2,2) \times S O(D-2)$ transformations, any null geodesic a distance $R$ from $\Sigma$ can be mapped to

$t=R u, \quad x^{1}=R u \cos \psi, \quad x^{2}=R u \sin \psi, \quad x^{3}=R$,

and $x^{i>3}=0$, where $\psi$ is the angle between $\Sigma$ and the null geodesic. Plugging Eqs. (3) and (4) into the ANEC gives

$$
\int_{-\infty}^{\infty} d u\left\langle T_{u u}\right\rangle=\frac{1}{6 \sqrt{\pi} R^{D}} \frac{|\sin \psi|}{\operatorname{vol}\left(\mathbb{S}^{D-3}\right)} \frac{\Gamma\left(\frac{D-1}{2}\right)}{\Gamma\left(\frac{D}{2}\right)} d_{2} \geq 0,
$$

which immediately implies $d_{2} \geq 0$.
EE and central charges.-Consider a CFT in D-dimensional Minkowski space with a flat 2D defect. We will compute the $\mathrm{EE}$ of a sphere of radius $\ell$ centered on the defect, using the method of Refs. [21,22].

We parametrize the Minkowski metric as

$$
\eta=-d t^{2}+\left(d x^{1}\right)^{2}+\left(d\left|x^{i}\right|\right)^{2}+\left|x^{i}\right|^{2} d s_{\mathbb{S}^{D-3}}^{2},
$$

with the defect along $t$ and $x^{1}$ and located at $\left|x^{i}\right|=0$. Defining $r^{2}=\left(x^{1}\right)^{2}+\left|x^{i}\right|^{2}$, the sphere's causal development is given by $r \pm t \leq \ell$. The change of coordinates

$$
\begin{aligned}
t & =\frac{\ell \cos \theta \sinh \left(\frac{\tau}{\ell}\right)}{1+\cos \theta \cosh \left(\frac{\tau}{\ell}\right)}, \quad r=\frac{\ell \sin \theta}{1+\cos \theta \cosh \left(\frac{\tau}{\ell}\right)}, \\
x^{1} & =r \cos \phi, \quad\left|x^{i}\right|=r \sin \phi .
\end{aligned}
$$

maps the sphere's causal development to the static patch of $D$-dimensional de Sitter space, $d S_{D}$, with metric

$$
\begin{aligned}
\Omega^{2} \eta & =-\ell^{-2} \cos ^{2} \theta d \tau^{2}+d \theta^{2}+\sin ^{2} \theta\left(d \phi^{2}+\sin ^{2} \phi d s_{\mathbb{S}^{D-3}}^{2}\right), \\
\Omega & =1+\cos \theta \cosh (\tau / \ell),
\end{aligned}
$$

with $\tau \in(-\infty, \infty), \theta \in[0, \pi / 2]$, and $\phi \in[0, \pi]$. The defect is then along $\tau$ and $\theta$ and is located at $\phi=0$, $\pi$, i.e., along a maximal $\mathrm{dS}_{2}$.

The reduced density matrix of the sphere's causal development maps to $e^{-\beta H_{\tau}}$ modulo normalization, with $\beta=2 \pi \ell$ and $H_{\tau}$ the generator of $\tau$ translations. As a result, $S_{\mathrm{EE}}$ maps to thermal entropy in $\mathrm{dS}_{D}$ at inverse temperature $\beta=2 \pi \ell, S_{\mathrm{EE}}=\beta E-F$, with $E$ the Killing energy corresponding to $H_{\tau}$ and $F=-\ln \operatorname{tr}\left(e^{-\beta H_{\tau}}\right)$ the dimensionless free energy. We define the defect's contribution as $S_{\mathrm{EE}}^{\mathrm{def}} \equiv S_{\mathrm{EE}}-S_{\mathrm{EE}}^{\mathrm{CFT}}$, with $S_{\mathrm{EE}}^{\mathrm{CFT}}$ the EE of a sphere of radius $\ell$ in the bulk CFT, with the same UV cutoff, and similarly for $E^{\text {def }}$ and $F^{\text {def }}$.

Analytically continuing to Euclidean time, $\tau=-i \tau_{E}$ with $\tau_{E} \sim \tau_{E}+\beta$, Eq. (8) becomes the metric of $\mathbb{S}^{D}$, with the defect wrapping a maximal $\mathbb{S}^{2}$. As a result, $F=-\ln Z$, with $Z$ the DCFT's Euclidean partition function on $\mathbb{S}^{D}$. Using $Z / Z_{\mathrm{CFT}} \propto(r \Lambda)^{b / 3}$ with $\Lambda=1 / \varepsilon$, we find

$$
F^{\mathrm{def}}=-\ln \left(Z / Z_{\mathrm{CFT}}\right)=-\frac{b}{3} \ln \left(\frac{\ell}{\varepsilon}\right)+\mathcal{O}\left(\varepsilon^{0}\right) .
$$

The defect's contribution to the Killing energy is

$$
E^{\mathrm{def}}=\int d S_{\mu} K_{\nu}\left\langle T^{\mu \nu}\right\rangle_{\mathrm{def}},
$$

with $\quad d S_{\mu}=\ell^{-1} \cos \theta(\sin \theta)^{D-2}(\sin \phi)^{D-3} d \theta d \phi d s_{\mathbb{S}_{D-3}} \delta_{\mu}^{\tau}$ the volume element on a constant time slice, and $K^{\mu} \partial_{\mu}=$ $\partial_{\tau}$ the time translation Killing vector. We thus need $\left\langle T^{\tau}{ }_{\tau}\right\rangle_{\text {def }}$, which we obtain by Weyl transformation from the Minkowski-space $\left\langle T^{\mu \nu}\right\rangle$ in Eq. (3), with the result 


$$
\left\langle T_{\tau}^{\tau}\right\rangle_{\mathrm{def}}=-\frac{(\sin \theta \sin \phi)^{-D}}{6 \pi \operatorname{vol}\left(\mathbb{S}^{D-3}\right)} \frac{D-3}{D-1} d_{2}-\frac{b}{24 \pi} \delta^{D-2},
$$

where the first term comes from Weyl-rescaling $T^{\mu \nu}$ and the second term comes from $T^{\mu \nu}$ 's anomalous Weyl transformation law. The integral of the second term is finite, but the integral of the first diverges at $\theta=0$ and the defect $\phi=0, \pi$. Using a regulator $\varepsilon / \ell$, we thus find

$$
\beta E^{\mathrm{def}}=-\frac{1}{3} \frac{D-3}{D-1} d_{2} \ln \left(\frac{\ell}{\varepsilon}\right)+\mathcal{O}\left(\varepsilon^{0}\right) .
$$

Plugging Eqs. (9) and (12) into $S_{\mathrm{EE}}$ then gives [41]

$$
S_{\mathrm{EE}}^{\mathrm{def}}=\frac{1}{3}\left(b-\frac{D-3}{D-1} d_{2}\right) \ln \left(\frac{\ell}{\varepsilon}\right)+\mathcal{O}\left(\varepsilon^{0}\right) .
$$

We have also derived Eq. (13) via the replica method, generalizing the result of Ref. [34] for 3D BCFTs to $D \geq 3$ DCFTs [44].

Further checking Eq. (13), we consider the holographic DCFT given by a probe brane along an $\mathrm{AdS}_{3}$ submanifold inside $\operatorname{AdS}_{D+1}$ of radius $L$, with action $S_{\text {probe }}$ above. In that case, Ref. [29] found

$$
S_{\mathrm{EE}}^{\mathrm{def}}=\frac{4 \pi T_{\mathrm{br}} L^{3}}{D-1} \ln \left(\frac{\ell}{\varepsilon}\right)+\mathcal{O}\left(\varepsilon^{0}\right),
$$

which agrees with Eq. (13), using $b$ and $d_{2}$ from Table I.

Equation (13) agrees with a key result of Ref. [23] that the coefficient of $\ln (\ell / \varepsilon)$ in $S_{\mathrm{EE}}^{\text {def }}$ includes a contribution from $\left\langle T_{\mu \nu}\right\rangle$, which we have shown is proportional $d_{2}$.

For defects of various dimensions, Refs. [23,45,46] found that the universal part of $S_{\mathrm{EE}}^{\text {def }}$ need not obey a defect $c$ theorem. For a 2D defect in a $D \geq 4$ CFT, Eq. (13) shows although $b$ obeys a defect $c$ theorem, the combination of $b$ and $d_{2}$ in Eq. (13) need not and does not [46].

Holographic examples.-First, we consider 4D $\mathcal{N}=4$ SUSY $U(N)$ YM theory at large $N$ and large 't Hooft coupling $\lambda$, dual to 10D type IIB supergravity (SUGRA) on $\mathrm{AdS}_{5} \times \mathbb{S}^{5}$ [47]. SUGRA solutions describing the most general 1/2-BPS 2D surface operators appear in Ref. [48]. Generically such a surface operator breaks $U(N) \rightarrow$ $\prod_{k=1}^{n} U\left(N_{k}\right)$ with $\sum_{k=1}^{n} N_{k}=N$ and produces a nonzero expectation value for one adjoint complex scalar field, $\Phi$, which decomposes into the block diagonal form

$$
\langle\Phi\rangle=\frac{e^{-i \phi}}{\sqrt{2}\left|x^{i}\right|} \operatorname{diag}\left(z_{1} \mathbb{1}_{N_{1}}, z_{2} \mathbb{1}_{2}, \ldots, z_{n} \mathbb{1}_{N_{n}}\right),
$$

with $\mathbb{S}^{1}$ angular coordinate $\phi$ around the defect, $z_{k} \in \mathbb{C}$ dimensionless parameters, and $\mathbb{1}_{N_{k}}$ the $N_{k} \times N_{k}$ identity matrix [48-51]. For such a defect Ref. [50] holographically computed $\left\langle T^{\mu \nu}\right\rangle$ for $\mathcal{M}=\mathrm{AdS}_{3} \times \mathbb{S}^{1}$, though the result is scheme dependent. We fix the scheme by conformally mapping $\mathrm{AdS}_{3} \times \mathbb{S}^{1}$ to Minkowski space and demanding that without a defect $\left\langle T^{\mu \nu}\right\rangle=0$. Reference [52] holographically computed $S_{\mathrm{EE}}^{\text {def }}$ for a sphere centered on the defect. Using these results, Eqs. (3) and (13) give

$$
\begin{aligned}
b & =3\left(N^{2}-\sum_{k=1}^{n} N_{k}^{2}\right), \\
d_{2} & =3\left(N^{2}-\sum_{k=1}^{n} N_{k}^{2}\right)+\frac{24 \pi^{2} N}{\lambda} \sum_{k=1}^{n} N_{k}\left|z_{k}\right|^{2} .
\end{aligned}
$$

Both of these are manifestly positive, and $b$ is independent of the marginal parameters $\lambda$ and $z_{k}$.

As discussed in Ref. [50] the one-loop $\left\langle T^{\mu \nu}\right\rangle$ on $\mathrm{AdS}_{3} \times \mathbb{S}^{1}$ in the presence of the surface operator matches the term $\propto N / \lambda$ in Eq. (16). Given that the other terms in Eq. (16) are independent of the marginal parameters at large $\lambda$, and that $T^{\mu \nu}$ on $\mathrm{AdS}_{3} \times \mathbb{S}^{1}$ is scheme dependent, $b$ and $d_{2}$ may in fact be one-loop exact.

Second, we study $1 / 2$-BPS Wilson surface defects in the 6D $A_{M-1} \mathcal{N}=(2,0)$ SUSY CFT specified by a representation, $\mathfrak{R}$, of $A_{M-1}$ with highest weight $w$ and a 2D surface [53]. When $M \gg 1$ the theory is holographically dual to 11D SUGRA on $\mathrm{AdS}_{7} \times \mathbb{S}^{4}$ [47], and Wilson surfaces are dual to M2 branes, or M5 branes with M2-brane flux, reaching the $\mathrm{AdS}_{7}$ boundary at $\Sigma$ [54-58].

Using the holographic results for $\left\langle T^{\mu \nu}\right\rangle$ and $S_{\mathrm{EE}}^{\mathrm{def}}$ in the presence of a flat Wilson surface [59,60], Eqs. (3) and (13) give

$b=24(w, \varrho)+3(w, w), \quad d_{2}=24(w, \varrho)+6(w, w)$,

where $\varrho$ is the gauge algebra's Weyl vector, and $(\cdot, \cdot)$ is the scalar product on the weight space. Both $b$ and $d_{2}$ are $\geq 0$ for all $\mathfrak{R}$ and are invariant under the action of the Weyl group. In the defect, RG flows triggered by the expectation value of a marginal Wilson surface operator studied holographically in Refs. [46,61,62], each of $b$ and $d_{2}$ is larger in the UV than in the IR, consistent with $b$ 's $c$ theorem.

Thermal entropy.-For a 2D CFT on $\mathbb{S}^{1}$ of radius $r$, in the thermodynamic limit $r T \rightarrow \infty, c$ determines the thermal entropy: $S=(\pi / 6) c T(2 \pi r)[63,64]$. Do $b, d_{1}$, and $d_{2}$ similarly determine a $2 \mathrm{D}$ boundary or defect's contribution to $S$ ?

Consider the 3D BCFTs of free, massless real scalar or Dirac fermion on a hemisphere of radius $r$. In the Supplemental Material [13], we calculate the boundary contribution to thermal entropy, $S_{\partial}$. When $r T \rightarrow \infty$, we find

$$
S_{\partial}^{R / D}= \pm \frac{\pi}{12} T(2 \pi r), \quad S_{\partial}^{\mathrm{f}}=0
$$

where the superscripts denote the Robin scalar, Dirichlet scalar, and Dirac fermion, respectively. Table I shows the 
Dirac fermion has $d_{1} \neq 0$, so $S_{\partial}^{\mathrm{f}}=0$ proves that $S_{\partial}$ cannot have a term $\propto d_{1}$ with universal nonzero coefficient. Instead, Table I and Eq. (18) suggest $S_{\partial} \stackrel{?}{=}(4 \pi / 3) b T(2 \pi r)$, which, if true, looks like eight times a Cardy entropy.

However, consider the holographic DCFT given by a probe brane along an asymptotically $\mathrm{AdS}_{3}$ submanifold inside an $\mathrm{AdS}_{D+1}$-Schwarzschild black hole of radius $L$ and temperature $T$, with action $S_{\text {probe }}$ above. In the Supplemental Material [13], we compute this defect's contribution to $S$

$$
S_{\mathrm{def}}=\frac{16 \pi^{2}}{D^{2}} L^{3} T_{\mathrm{br}} T(2 \pi r),
$$

which via Table I we can write as $S_{\text {def }}=$ ? $\left(1 / D^{2}\right)(8 \pi / 3) b T(2 \pi r)$, but without further input this choice is arbitrary as $b=d_{1}=d_{2}$ [30]. We can compare to a DCFT given by gluing two free-field 3D BCFTs along their boundaries, with no boundary interactions, whose $S_{\text {def }}$ is simply a sum of the $S_{\partial}$ in Eq. (18). When $D=3$, no such sum can produce the $1 / D^{2}=1 / 9$ factor in the holographic $S_{\text {def }}$. This proves that if $S_{\text {def }} \propto b T(2 \pi r)$, then the coefficient cannot be universal.

We are pleased to thank M. Buican, A. Chalabi, N. Drukker, T. Hartman, T. Faulkner, and J. Sisti for helpful discussions. We especially thank J. Estes, C. Herzog, D. Krym, and M. Gutperle for comments on the draft. K. J. is supported in part by the Department of Energy under Award No. DE-SC0013682. A. O'B. is a Royal Society University Research Fellow. B. R. and R. R. acknowledge support from STFC through consolidated Grant No. ST/ L000296/1.

*kristanj@sfsu.edu

†a.obannon@soton.ac.uk

*B.J.Robinson@soton.ac.uk

${ }^{\S}$ R.J.Rodgers@soton.ac.uk

[1] A. Zamolodchikov, Pis'ma Zh. Eksp. Teor. Fiz. 43, 565 (1986).[JETP Lett. 43, 730 (1986)].

[2] J. Wess and B. Zumino, Phys. Lett. 37B, 95 (1971).

[3] L. Bonora, P. Pasti, and M. Bregola, Classical Quantum Gravity 3, 635 (1986).

[4] S. Deser and A. Schwimmer, Phys. Lett. B 309, 279 (1993).

[5] M. J. Duff, Classical Quantum Gravity 11, 1387 (1994).

[6] C. Holzhey, F. Larsen, and F. Wilczek, Nucl. Phys. B424, 443 (1994).

[7] P. Calabrese and J. Cardy, J. Stat. Mech. (2004) P06002.

[8] J. L. Cardy, Nucl. Phys. B270, 186 (1986).

[9] I. Affleck, Phys. Rev. Lett. 56, 746 (1986).

[10] Every independently conserved spin-2 operator at the boundary or defect may give rise to a Virasoro symmetry. However, in general, such operators appear only if the 2D degrees of freedom decouple from the bulk CFT, or if the bulk CFT has no propagating degrees of freedom. An example of the latter is 3D Chern-Simons theory, which is topological and hence has vanishing stress tensor.

[11] M. Henningson and K. Skenderis, J. High Energy Phys. 06 (1999) 012.

[12] A. Schwimmer and S. Theisen, Nucl. Phys. B801, 1 (2008).

[13] See Supplemental Material at http://link.aps.org/ supplemental/10.1103/PhysRevLett.122.241602 for the description of parity violating contributions to the Weyl anomaly of a 2D surface defect in a 4D CFT and computation of thermal entropy for 2D free field BCFTs and holographic probe brane DCFTs, which includes Refs. [14-20].

[14] J. A. Harvey and A. B. Royston, J. High Energy Phys. 04 (2008) 018.

[15] E. I. Buchbinder, J. Gomis, and F. Passerini, J. High Energy Phys. 12 (2007) 101.

[16] J. A. Harvey and A. B. Royston, J. High Energy Phys. 08 (2008) 006.

[17] S. Giombi, I. R. Klebanov, and A. A. Tseytlin, Phys. Rev. D 90, 024048 (2014).

[18] P. van Nieuwenhuizen, in Strings, Gauge Fields, and the Geometry Behind: The Legacy of Maximilian Kreuzer, edited by A. Rebhan, L. Katzarkov, J. Knapp, R. Rashkov, and E. Scheidegger (World Scientific, Singapore, 2012), pp. 133-157.

[19] C. Csaki, C. Grojean, J. Hubisz, Y. Shirman, and J. Terning, Phys. Rev. D 70, 015012 (2004).

[20] E. Witten, Adv. Theor. Math. Phys. 2, 505 (1998).

[21] H. Casini, M. Huerta, and R. Myers, J. High Energy Phys. 05 (2011) 036.

[22] K. Jensen and A. O'Bannon, Phys. Rev. D 88, 106006 (2013).

[23] N. Kobayashi, T. Nishioka, Y. Sato, and K. Watanabe, J. High Energy Phys. 01 (2019) 039.

[24] In the replica trick approach to calculating Rényi entropies in a 4D CFT, twist operators appear as 2D conformal defects defined by boundary conditions on bulk CFT fields. Those defect's central charges can then determine both universal [25-27] and nonuniversal [28] terms in EE.

[25] A. Lewkowycz and E. Perlmutter, J. High Energy Phys. 01 (2015) 080.

[26] E. Perlmutter, M. Rangamani, and M. Rota, Phys. Rev. Lett. 115, 171601 (2015).

[27] L. Bianchi, M. Meineri, R. C. Myers, and M. Smolkin, J. High Energy Phys. 07 (2016) 076.

[28] K. Ohmori and Y. Tachikawa, J. Stat. Mech. (2015) P04010.

[29] K. Jensen and A. O'Bannon, Phys. Rev. Lett. 116, 091601 (2016).

[30] C. R. Graham and E. Witten, Nucl. Phys. B546, 52 (1999).

[31] C. P. Herzog and K.-W. Huang, J. High Energy Phys. 10 (2017) 189.

[32] C. Herzog, K.-W. Huang, and K. Jensen, Phys. Rev. Lett. 120, 021601 (2018).

[33] M. Nozaki, T. Takayanagi, and T. Ugajin, J. High Energy Phys. 06 (2012) 066.

[34] D. V. Fursaev and S. N. Solodukhin, Phys. Rev. D 93, 084021 (2016).

[35] Every independently conserved spin-2 operator at the boundary, with associated central charge $c$, contributes a term $\propto c$ to $b$ in addition to those in Eq. (2). 
[36] D. McAvity and H. Osborn, Nucl. Phys. B406, 655 (1993).

[37] D. McAvity and H. Osborn, Nucl. Phys. B455, 522 (1995).

[38] T. Faulkner, R. G. Leigh, O. Parrikar, and H. Wang, J. High Energy Phys. 09 (2016) 038.

[39] T. Hartman, S. Kundu, and A. Tajdini, J. High Energy Phys. 07 (2017) 066.

[40] A. Kapustin, Phys. Rev. D 74, 025005 (2006).

[41] These arguments can be generalized to the EE of a hemisphere of radius $r$ ending on the boundary of a 4D BCFT. We find that the boundary's contribution is equal to minus Gaiotto's "boundary $\mathcal{F}$ " [42]. Evidence from holography [43] and free fields [42] suggests that $\mathcal{F}$ obeys a boundary $c$ theorem.

[42] D. Gaiotto, arXiv:1403.8052.

[43] J. Estes, K. Jensen, A. O'Bannon, E. Tsatis, and T. Wrase, J. High Energy Phys. 05 (2014) 084.

[44] Reference [34] also considered EE of regions that do not intersect the boundary perpendicularly, finding a logarithmic term whose coefficient is a linear combination of $b$ and $d_{1}$. However, in such cases, the replica symmetry is only $\mathbb{Z}_{n}$ with replica index $n$, in contrast to a CFT or a region that hits a boundary or defect perpendicularly, where $\mathbb{Z}_{n}$ is enhanced to $U(1)$. The analytic continuation required to compute $S_{\mathrm{EE}}$, from integer $n$ to $n=1+\delta$ with $\delta \ll 1$, is more delicate with only the $\mathbb{Z}_{n}$ symmetry.

[45] S. P. Kumar and D. Silvani, J. High Energy Phys. 01 (2018) 052.

[46] R. Rodgers, J. High Energy Phys. 03 (2019) 092.

[47] J. M. Maldacena, Adv. Theor. Math. Phys. 2, 231 (1998).
[48] J. Gomis and S. Matsuura, J. High Energy Phys. 06 (2007) 025.

[49] S. Gukov and E. Witten, arXiv:hep-th/0612073.

[50] N. Drukker, J. Gomis, and S. Matsuura, J. High Energy Phys. 10 (2008) 048.

[51] N. Drukker, J. Gomis, and D. Young, J. High Energy Phys. 03 (2009) 004.

[52] S. A. Gentle, M. Gutperle, and C. Marasinou, J. High Energy Phys. 04 (2016) 067.

[53] O. J. Ganor, Nucl. Phys. B489, 95 (1997).

[54] O. Lunin, J. High Energy Phys. 10 (2007) 014.

[55] B. Chen, W. He, J.-B. Wu, and L. Zhang, J. High Energy Phys. 08 (2007) 067.

[56] E. D'Hoker, J. Estes, M. Gutperle, and D. Krym, J. High Energy Phys. 12 (2008) 044.

[57] C. Bachas, E. D'Hoker, J. Estes, and D. Krym, Fortschr. Phys. 62, 207 (2014).

[58] H. Mori and S. Yamaguchi, Phys. Rev. D 90, 026005 (2014).

[59] S. A. Gentle, M. Gutperle, and C. Marasinou, J. High Energy Phys. 08 (2015) 019.

[60] J. Estes, D. Krym, A. O'Bannon, B. Robinson, and R. Rodgers, J. High Energy Phys. 05 (2019) 032.

[61] J. Camino, A. Paredes, and A. Ramallo, J. High Energy Phys. 05 (2001) 011.

[62] J. Gomis, A. V. Ramallo, J. Simon, and P. K. Townsend, J. High Energy Phys. 11 (1999) 019.

[63] J. L. Cardy, J. Phys. A 17, L385 (1984).

[64] I. Affleck, Phys. Rev. Lett. 56, 746 (1986). 